\title{
State and Unknown Input Simultaneous Estimation for a Class of Discrete- Time Linear Implicit Models : A Heat Exchanger Pilot Process Application
}

\author{
Karim Bouassem ${ }^{1,2, *}$, El Mahfoud El Bouatmani ${ }^{1,2, * *}$, Abdellatif El Assoudi ${ }^{1,2, * * *}$, and El Hassane El Yaagoubi ${ }^{1,2, * * * *}$ \\ ${ }^{1}$ Laboratory of High Energy Physics and Condensed Matter, Faculty of Science, Hassan II University of Casablanca. \\ B.P 5366, Maarif, Casablanca, Morocco. \\ ${ }^{2}$ ECPI, Department of Electrical Engineering, ENSEM, Hassan II University of Casablanca. \\ B.P 8118, Oasis, Casablanca, Morocco.
}

\begin{abstract}
In this paper, the design problem of simultaneous estimation of unmeasurable states and unknown inputs (UIs) is investigated for a class of discrete-time linear implicit models (DLIMs). The UIs affect both state and output of the system. The approach is based on the separation between dynamic and static relations in the considered DLDM. First, the method permitting to separate dynamic equations from static equations is exposed. Next, an augmented explicit model which contains the dynamic equations and the UIs is constructed. Then an unknown inputs observer (UIO) design in explicit structure is developed. The exponential convergence of the state estimation error is studied by using the Lyapunov theory and the stability condition is given in term of linear matrix inequality (LMI). Finally, an illustrative application of a heat exchanger pilot process is given to show the good performances of the proposed method.
\end{abstract}

\section{Introduction and problem statement}

\subsection{Introduction}

Nowadays, one of the goals of many industries is to maintain the best process performance in terms of efficiency, reliability, availability, safety and security. Thus, it has become so necessary to equip industrial processes with physical sensors in order to collect all the information necessary to discover any unexpected change that may occur in the process. In this perspective, it is well-known that the algorithm called an observer permits to provide on-line estimation of unmeasurable states of various industrial processes. This last theme which the method consists to combine a priori knowledge about a process with experimental data, has attracted much attention of the researchers for a long time. This is due to its important role in the control and fault-tolerant areas.

Otherwise, UIs can be result either from uncertainty in the model or from the presence of unknown external excitation. Thus, the UIO design largely used in the area of fault detection and diagnosis and design of fault tolerant control strategy has received considerable attention and is one of the most attractive research areas during these last two decades. Indeed, many research works based on different approaches exist in the literature [1-7].

\footnotetext{
*e-mail: karim.bouassem@gmail.com

**e-mail: e.elbouatmani@ensem.ac.ma

***e-mail: a.elassoudi@ensem.ac.ma

****e-mail: h.elyaagoubi@ensem.ac.ma
}

Too, it is well known that implicit models also called descriptor systems, singular models or differential-algebraic equations (DAEs) have been widely used as a powerful modeling tool of to describe the behavior of many chemical and physical processes. They represent physical phenomena that can not be described by standard explicit models. We may cite [8-10] for some real applications of implicit models. The numerical simulation of such models usually combines an ordinary differential equation (ODE) numerical method together with an optimization algorithm.

The aim of this paper is the development of an UIO for a class of DLIMs. Indeed, many research works on observer design and its application to fault detection and diagnosis exist in the literature. They concern explicit and singular linear models in both continuous and discrete time cases see e.g. [11-22].

The main contribution of this paper is to give an UIO design for a class of DLIMs allowing the simultaneous estimation of the unknown states and UIs. A new design methodology through judicious use of the separation between the recurrent and static relations in the implicit model is proposed. Based on the Lyapunov theory, the exponential stability condition of the UIO is given in term of LMI [23].

Besides, for reasons of ease of the implementation, the main result of this paper consists in showing that the UIO problem for the considered class of DLIMs can be 
achieved by using an observer having only an explicit structure.

Throughout this paper we take up the following notations: $A^{T}$ is the transpose of matrix $A$. $A>0$ means that matrix $A$ is symmetric and positive definite.

The symbol $I$ (or 0 ) represents the identity matrix (or zero matrix) with the appropriate dimension.

\subsection{Problem statement}

In this paper, the following class of DLIMs subject to UIs which affect both state and output of the system is considered:

$$
\left\{\begin{array}{ccc}
\Lambda X_{k+1} & =\Sigma X_{k}+\Gamma U_{k}+\Phi \eta_{k} \\
Y_{k} & =\Omega X_{k}+\Pi U_{k}+\Psi \eta_{k}
\end{array}\right.
$$

where

$X_{k}^{T}=\left[X_{k}^{1^{T}} X_{k}^{2^{T}}\right] \in \mathbf{R}^{n}$ is the state vector.

$X_{k}^{1} \in \mathbf{R}^{n_{1}}$ is the vector of recurrent variables.

$X_{k}^{2} \in \mathbf{R}^{n_{2}}$ is the vector of algebraic variables. $n_{1}+n_{2}=n$.

$U_{k} \in \mathbf{R}^{m}$ is the control input.

$Y_{k} \in \mathbf{R}^{p}$ is the measured output.

$\eta_{k} \in \mathbf{R}^{r}$ is the unknown input.

$\Sigma \in \mathbf{R}^{n \times n}, \Gamma \in \mathbf{R}^{n \times m}, \Omega \in \mathbf{R}^{p \times n}, \Phi \in \mathbf{R}^{n \times r}, \Pi \in \mathbf{R}^{p \times m}$, $\Psi \in \mathbf{R}^{p \times r}, \Lambda \in \mathbf{R}^{n \times n}$ such that $\operatorname{rank}(\Lambda)=n_{1}$ are real known constant matrices with:

$$
\begin{aligned}
& \Lambda=\left(\begin{array}{cc}
I & 0 \\
0 & 0
\end{array}\right) ; \quad \Sigma=\left(\begin{array}{cc}
\Sigma_{11} & \Sigma_{12} \\
\Sigma_{21} & \Sigma_{22}
\end{array}\right) \\
& \Gamma=\left(\begin{array}{c}
\Gamma_{1} \\
\Gamma_{2}
\end{array}\right) ; \Phi=\left(\begin{array}{c}
\Phi_{1} \\
\Phi_{2}
\end{array}\right) ; \Omega=\left(\begin{array}{ll}
\Omega_{1} & \Omega_{2}
\end{array}\right)
\end{aligned}
$$

where constant matrices $\Sigma_{22}$ are supposed invertible.

Before giving the main result, let us make the following assumption :

\section{Assumption 1 Suppose that [8]:}

- $(\Lambda, \Sigma)$ is regular, i.e. $\operatorname{det}(z \Lambda-\Sigma) \neq 0 \forall z \in \mathbf{C}$

- The model (1) is impulse observable and detectable.

In order to investigate the UIO design for system (1), we proceed as mentioned above to the separation of the recurrent equations from static equations of the model (1). Indeed, using (2) and (3) the system (1) can be rewritten as follows:

$$
\left\{\begin{array}{cl}
X_{k+1}^{1} & =\Sigma_{11} X_{k}^{1}+\Sigma_{12} X_{k}^{2}+\Gamma_{1} U_{k}+\Phi_{1} \eta_{k} \\
0 & =\Sigma_{21} X_{k}^{1}+\Sigma_{22} X_{k}^{2}+\Gamma_{2} U_{k}+\Phi_{2} \eta_{k} \\
Y_{k} & =\Omega_{1} X_{k}^{1}+\Omega_{2} X_{k}^{2}+\Pi U_{k}+\Psi \eta_{k}
\end{array}\right.
$$

The form (4) for system (1) is also known as the second equivalent form.

Since $\Sigma_{22}$ is inversible, it follows:

$$
X_{k}^{2}=J X_{k}^{1}+K U_{k}+L \eta_{k}
$$

where

$$
\left\{\begin{array}{l}
J=-\Sigma_{22}^{-1} \Sigma_{21} \\
K=-\Sigma_{22}^{-1} \Gamma_{2} \\
L=-\Sigma_{22}^{-1} \Phi_{2}
\end{array}\right.
$$

Thus, combining (4) and (5) we have:

$$
\left\{\begin{array}{c}
X_{k+1}^{1}=M X_{k}^{1}+N U_{k}+P \eta_{k} \\
X_{k}^{2}=J X_{k}^{1}+K U_{k}+L \eta_{k} \\
Y_{k}=R X_{k}^{1}+S U_{k}+T \eta_{k}
\end{array}\right.
$$

where

$$
\left\{\begin{array}{l}
M=\Sigma_{11}+\Sigma_{12} J \\
N=\Gamma_{1}+\Sigma_{12} K \\
P=\Phi_{1}+\Phi_{12} L \\
R=\Omega_{1}+\Omega_{2} J \\
S=\Pi+\Omega_{2} K \\
T=\Psi+\Omega_{2} L
\end{array}\right.
$$

Assumption 2 : Suppose that $\eta_{k}$ is considered as a constant unknown input per time interval i.e.:

$$
\eta_{k+1}=\eta_{k} \quad k \in\left[\begin{array}{ll}
T_{1} & T_{2}
\end{array}\right], \quad \forall T_{1}, T_{2} \in \mathbf{R}^{+}
$$

Let us define the augmented state vector $\xi_{k}^{1}=\left[\begin{array}{ll}X_{k}^{1} & \eta_{k}^{T}\end{array}\right]^{T}$ and $\xi_{k}^{2}=X_{k}^{2}$. Thus, the system (7) can be represented as:

$$
\left\{\begin{array}{cc}
\xi_{k+1}^{1} & =\tilde{M} \xi_{k}^{1}+\tilde{N} U_{k} \\
\xi_{k}^{2} & =\tilde{J} \xi_{k}^{1}+K U_{k} \\
Y_{k} & =\tilde{R} \xi_{k}^{1}+S U_{k}
\end{array}\right.
$$

where

$$
\left\{\begin{aligned}
\tilde{M} & =\left(\begin{array}{cc}
M & P \\
0 & I
\end{array}\right) \\
\tilde{N} & =\left(\begin{array}{c}
N \\
0
\end{array}\right) \\
\tilde{J} & =\left(\begin{array}{cc}
J & L \\
R & T
\end{array}\right)
\end{aligned}\right.
$$

The rest of the paper is structured as follows. The main result about UIO design permitting to estimate simultaneously unmeasurable states and UIs for the considered class of DLIMs (1) is stated in Section 2. The observer gain is found directly from LMI formulation. In Section 3, a heat exchanger pilot process application to show the good performance of the proposed technique is presented. Finally, a conclusion is given in section 4 .

\section{State and unknown inputs estimation}

Models (1) and (10) are equivalent. For the design of the UIO permitting the simultaneous estimation of the unmeasurable states and UIs, we will use the second structure. So, the proposed UIO takes the following form:

$$
\left\{\begin{array}{cl}
\hat{\xi}_{k+1}^{1} & =\tilde{M} \hat{\xi}_{k}^{1}+\tilde{N} U_{k}-G\left(\hat{Y}_{k}-Y_{k}\right) \\
\hat{\xi}_{k}^{2} & =\tilde{J} \hat{\xi}_{k}^{1}+K U_{k} \\
\hat{Y}_{k} & =\tilde{R} \hat{\xi}_{k}^{1}+S U_{k}
\end{array}\right.
$$

where $\left(\hat{\xi}_{k}^{1}, \hat{\xi}_{k}^{2}\right)$ and $\hat{Y}_{k}$ denote the estimated augmented state vector and the output vector respectively. $G$, is 
the gains of UIO which is determined such that $\left(\hat{\xi}_{k}^{1}, \hat{\xi}_{k}^{2}\right)$ asymptotically converges to $\left(\xi_{k}^{1}, \xi_{k}^{2}\right)$.

In order to establish the condition for the exponential convergence of the observer (12), we define the state estimation error:

$$
\varepsilon_{k}=\left(\begin{array}{c}
\varepsilon_{k}^{1} \\
\varepsilon_{k}^{2}
\end{array}\right)=\left(\begin{array}{c}
\hat{\xi}_{k}^{1}-\xi_{k}^{1} \\
\hat{\xi}_{k}^{2}-\xi_{k}^{2}
\end{array}\right)
$$

It follows from (10) and (12) that the estimated error equation can be written as:

$$
\left\{\begin{array}{c}
\varepsilon_{k+1}^{1}=H \varepsilon_{k}^{1} \\
\varepsilon_{k}^{2}=\tilde{J} \varepsilon_{k}^{1}
\end{array}\right.
$$

where

$$
H=\tilde{M}-G \tilde{R}
$$

To prove the convergence of the estimation error $\varepsilon_{k}$ toward zero, it suffices to prove from (14), that $\varepsilon_{k}^{1}$ converges toward zero. The main result is stated in the following Theorem.

Theorem 1 : There exists an UIO (12) for DLIM (1) if given $0<\alpha<1$ there exist matrices $Q>0$, W verifying the following LMI:

$$
\left.\left(\begin{array}{cc}
-\alpha^{2} Q & * \\
Q \tilde{M}-W \tilde{R} & -Q
\end{array}\right)<0 \quad \forall\right\}
$$

The observer gain $G$ is given by:

$$
G=Q^{-1} W
$$

Proof of Theorem 1 : Let us consider the following quadratic Lyapunov function as follows:

$$
V_{k}=\left(\varepsilon_{k}^{1}\right)^{T} Q \varepsilon_{k}^{1}, \quad Q>0
$$

Estimation error convergence is exponentially ensured if the following condition is guaranteed :

$$
\begin{aligned}
V_{k+1}-V_{k} & =\left(\varepsilon_{k+1}^{1}\right)^{T} Q \varepsilon_{k+1}^{1}-\left(\varepsilon_{k}^{1}\right)^{T} Q \varepsilon_{k}^{1} \\
& <\left(\alpha^{2}-1\right) V_{k}
\end{aligned}
$$

with $0<\alpha<1$.

By using (14), the condition (19) can be written as:

$$
\begin{gathered}
V_{k+1}-V_{k}=\left(\varepsilon_{k}^{1}\right)^{T}\left(H^{T} Q H-Q\right) \varepsilon_{k}^{1} \\
<\left(\alpha^{2}-1\right) V_{k}
\end{gathered}
$$

which is equivalent to the following stability condition:

$$
H^{T} Q H-\alpha^{2} Q<0
$$

Thus, by setting $W=Q G$, from (15) it follows that (21) is equivalent to (16) by using the Schur complement [23]. Finally, from the Lypunov stability theory, if the LMI condition (16) is satisfied, the error dynamic equation (14) is exponentially asymptotically stable.

\section{Application to a heat exchanger pilot process}

In order to demonstrate the effectiveness and applicability of the proposed UIO design approach with the aim of simultaneous estimation in real time of the unknown states and UIs, let us consider the heat exchanger pilot process described by the following Figure 1:

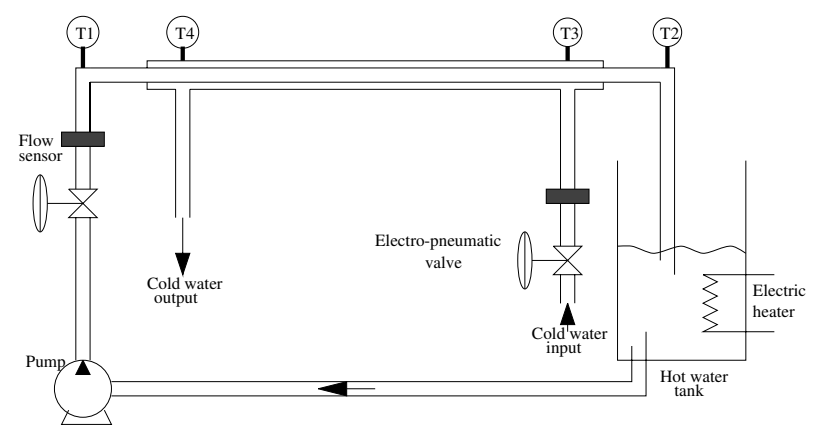

Figure 1. Heat exchanger process.

In this illustrative application, the following DLIM that we consider here to describe the behavior of the considered process assumed to be affected by an unknown input, is obtained by Euler discretization with a sampling period $\tau=0.012 s$ of the continuous-time linear implicit model given in [22]. It takes the form:

$$
\left\{\begin{array}{ccc}
\Lambda X_{k+1} & =\Sigma X_{k}+\Gamma U_{k}+\Phi \eta_{k} \\
Y_{k} & =\Omega X_{k}+\Pi U_{k}+\Psi \eta_{k}
\end{array}\right.
$$

where

$X_{k}^{T}=\left[X_{k}^{1^{T}} X_{k}^{2^{T}}\right] \in \mathbf{R}^{8}, X_{k}^{1}=\left[X_{1 k}^{1} \ldots X_{6 k}^{1}\right]^{T} \in \mathbf{R}^{6}$,

$X_{k}^{2}=\left[X_{7 k}^{1} X_{8 k}^{1}\right]^{T} \in \mathbf{R}^{2}, U_{k} \in \mathbf{R}^{2}, Y_{k} \in \mathbf{R}^{2}, \eta_{k} \in \mathbf{R}$.

As mentioned in (2)-(3), the sub-matrices of $\Sigma, \Gamma, \Omega, \Pi, \Lambda$ and $\Phi, \Psi$ have the following expressions:

$$
\begin{gathered}
\Lambda=\left(\begin{array}{ll}
I & 0 \\
0 & 0
\end{array}\right) \text { with } \operatorname{rank}(\Lambda)=6 \\
\Sigma_{11}=\left(\begin{array}{cccccc}
0.9863 & 66.3104 & 0 & 0.0034 & 0 & 0 \\
0 & 1.0000 & 0.0120 & 0 & 0 & 0 \\
0 & 0 & 1.0000 & 0 & 0 & 0 \\
0.0011 & 0 & 0 & 0.9971 & -20.9983 & 0 \\
0 & 0 & 0 & 0 & 1.0000 & 0.0120 \\
0 & 0 & 0 & 0 & 0 & 1.0000
\end{array}\right) \\
\Sigma_{21}=\left(\begin{array}{ccccccc}
0 & -0.4737 & -0.1056 & 0 & 0 & 0 \\
0 & 0 & 0 & 0 & -0.4737 & -0.1056
\end{array}\right)
\end{gathered}
$$$$
\Sigma_{12}=\left(\begin{array}{cc}
0 & 0 \\
0 & 0 \\
0.0120 & 0 \\
0 & 0 \\
0 & 0 \\
0 & 0.0120
\end{array}\right) ; \quad \Sigma_{22}=\left(\begin{array}{cc}
-0.0120 & 0 \\
0 & -0.0120
\end{array}\right)
$$$$
\Omega_{1}=\left(\begin{array}{cccccc}
1 & 0 & 0 & 0 & 0 & 0 \\
0 & 0 & 0 & 1 & 0 & 0
\end{array}\right) ; \quad \Omega_{2}=\left(\begin{array}{cc}
0 & 0 \\
0 & 0
\end{array}\right)
$$ 


$$
\begin{gathered}
\Gamma_{1}=\left(\begin{array}{ll}
0 & 0 \\
0 & 0 \\
0 & 0 \\
0 & 0 \\
0 & 0 \\
0 & 0
\end{array}\right) ; \Phi_{1}=\left(\begin{array}{c}
0.0024 \\
0.0003 \\
0 \\
0.0024 \\
0.0054 \\
0
\end{array}\right) ; \Pi=\left(\begin{array}{cc}
0 & 0 \\
0 & 0
\end{array}\right) \\
\Gamma_{2}=\left(\begin{array}{cc}
0.4406 & 0 \\
0 & 0.4406
\end{array}\right) ; \Phi_{2}=\left(\begin{array}{l}
0 \\
0
\end{array}\right) ; \Psi=\left(\begin{array}{l}
0 \\
0
\end{array}\right)
\end{gathered}
$$

Since $\Sigma_{22}$ is invertible, the DLIM (18) can be rewritten in the following equivalent system:

$$
\left\{\begin{array}{c}
X_{k+1}^{1}=M X_{k}^{1}+N U_{k}+P \eta_{k} \\
X_{k}^{2}=J X_{k}^{1}+K U_{k}+L \eta_{k} \\
Y_{k}=R X_{k}^{1}+S U_{k}+T \eta_{k}
\end{array}\right.
$$

where $M, N, P, J, K, L$ can be calculated by using (6)-(8).

Let us define the augmented state vector $\xi_{k}^{1}=\left[\begin{array}{ll}X_{k}^{1 T} & \eta_{k}^{T}\end{array}\right]^{T}$ and $\xi_{k}^{2}=X_{k}^{2}$.

Thus, under Assumption 2 (see Figure 6), the system (23) can be rewritten as:

$$
\left\{\begin{array}{c}
\xi_{k+1}^{1}=\tilde{M} \xi_{k}^{1}+\tilde{N} U_{k} \\
\xi_{k}^{2}=\tilde{J} \xi_{k}^{1}+K U_{k} \\
Y_{k}=\tilde{R} \xi_{k}^{1}+S U_{k}
\end{array}\right.
$$

where $\tilde{M}, \tilde{N}, \tilde{J}, \tilde{R}$ can be calculated by using (11).

By Theorem 1, considering $\alpha=0.9$ the following observer gain $G$ is obtained:

$$
G=\left(\begin{array}{rr}
1.9903 & -0.1139 \\
0.0151 & -0.0033 \\
-0.0105 & 0.0014 \\
-0.0097 & 2.5892 \\
0.0010 & -0.0990 \\
-0.0006 & 0.0666 \\
0.0406 & -2.2204
\end{array}\right)
$$

Simulation results with initial conditions:

$\xi_{0}^{1}=\left[\begin{array}{lllllll}10.0000 & -0.0016 & 0 & -19.0000 & -0.0016 & 0 & 0.1000\end{array}\right]^{T}$

$\hat{\xi}_{0}^{1}=\left[\begin{array}{lllllll}10.0000 & 0.0004 & 0.0020 & -19.0000 & 0.0004 & 0.0020 & 0.350\end{array}\right.$

$\xi_{0}^{2}=\left[\begin{array}{ll}0.3671 & 0.0734\end{array}\right]^{T} ; \hat{\xi}_{0}^{2}=\left[\begin{array}{ll}0.2706 & -0.0231\end{array}\right]^{T}$

are given in Figure 2 to Figure 6.

These simulation results show the performances of the proposed UIO (12) with the gains $G$, where the dashed lines denote the state variables and unknown input estimated by the observer. They show that the observer gives a good estimation of unmeasurable states and UI of the considered heat exchanger pilot process.
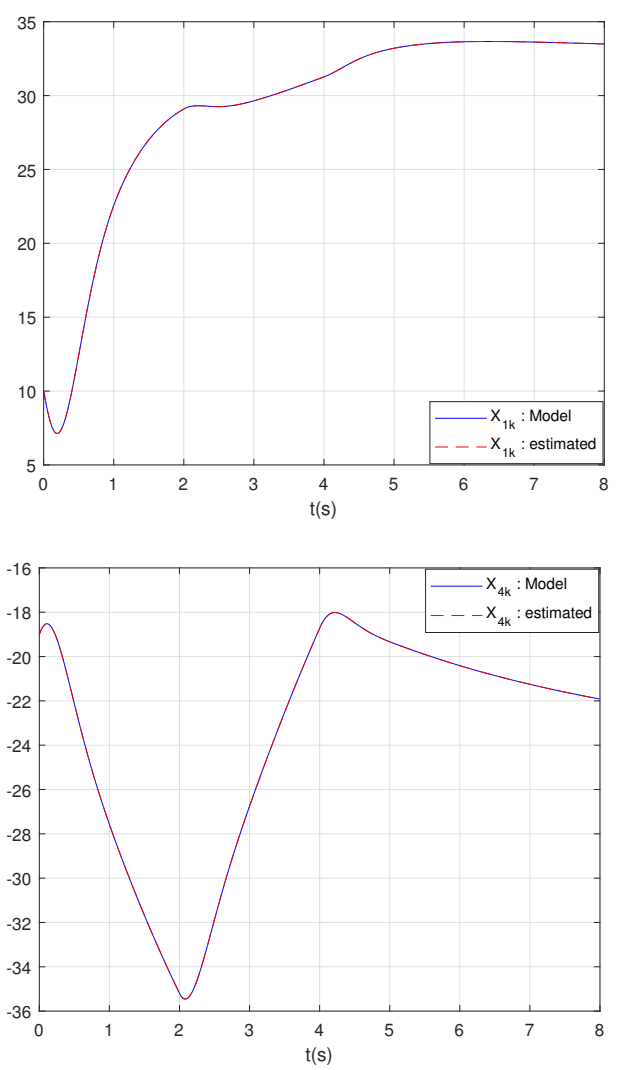

Figure 2. $X_{1 k}$ and $X_{4 k}$ with their estimates
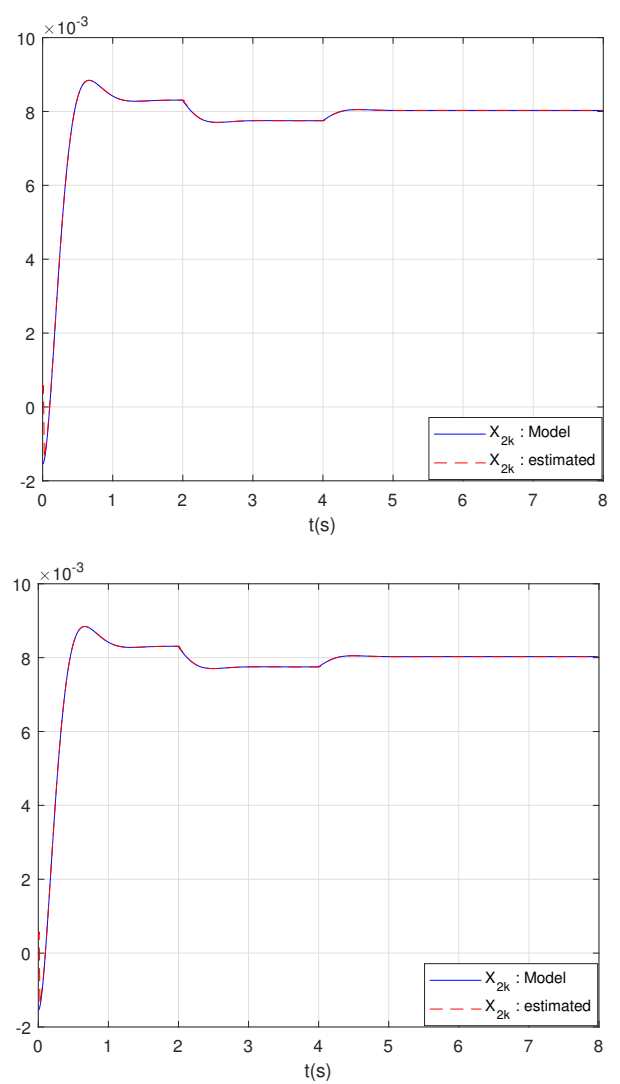

Figure 3. $X_{2 k}$ and $X_{5 k}$ with their estimates 

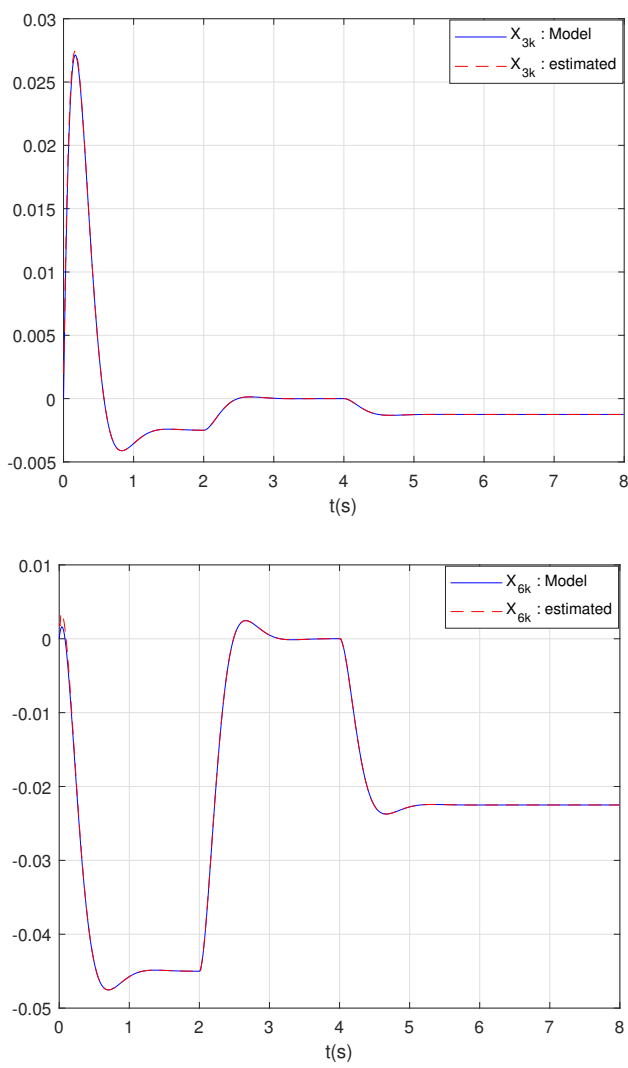

Figure 4. $X_{3 k}$ and $X_{6 k}$ with their estimates
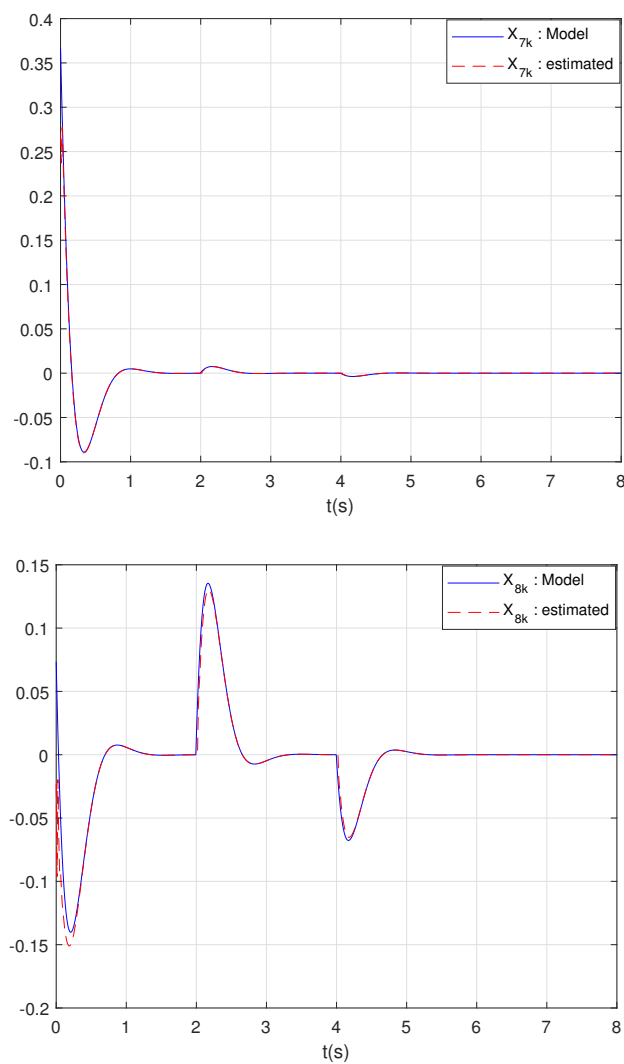

Figure 5. $X_{7 k}$ and $X_{8 k}$ with their estimates

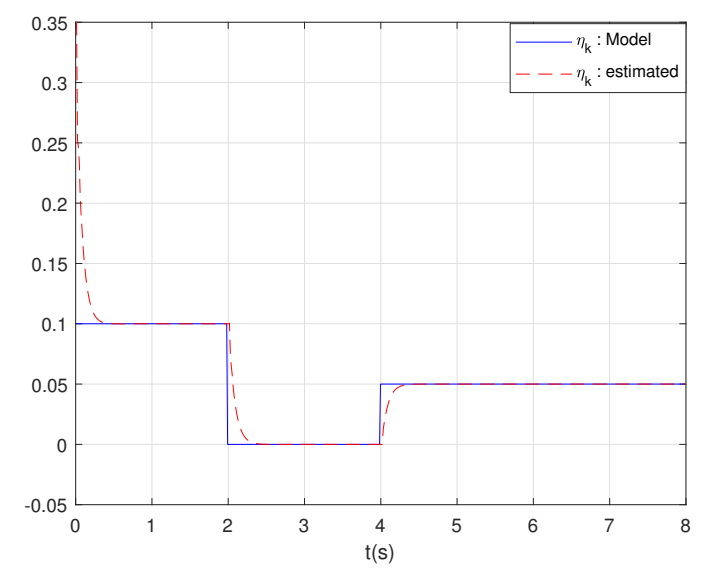

Figure 6. Unknown input $\eta_{k}$ with its estimate

\section{Conclusion}

A new result of UIO design permitting to estimate simultaneously the unmeasurable states and the UIs for a class of DLIMs is proposed in this paper. The main idea based on the second equivalent form of the linear implicit model which leads to the separation between the dynamic and static relations of the implicit model and the construction of an explicit structure formed by the dynamic equations and the UIs. The exponential convergence of the state estimation error is studied by using the Lyapunov theory and the stability condition is given in term of LMI. Simulation results are given and demonstrate the good performance of the proposed UIO design.

\section{References}

[1] R. Isermann, Fault-Diagnosis Systems An Introduction from Fault Detection to Fault Tolerance. Springer-Verlag Berlin Heidelberg 2006.

[2] Z. Lendek, T. M. Guerra, R. Babuška and B. De Schutter, Stability Analysis and Nonlinear Observer Design Using Takagi-Sugeno Fuzzy Models. Springer-Verlag Berlin Heidelberg 2010.

[3] R. Isermann, Fault-Diagnosis Applications, ModelBased Condition Monitoring: Actuators, Drives, Machinery, Plants, Sensors, and Fault-tolerant Systems, Springer-Verlag Berlin Heidelberg (2011). https://doi.org/10.1007/978-3-642-12767-0_2.

[4] M. Blanke, M. Kinnaert, J. Lunze, M. Staroswiecki, Diagnosis and Fault-Tolerant Control. SpringerVerlag Berlin Heidelberg (2016). https://doi.org/10. 1007/978-3-662-47943-8.

[5] L. Li, Fault Detection and Fault-Tolerant Control for Nonlinear Systems. Springer Fachmedien Wiesbaden 2016.

[6] L. Ming, J. Zhao, Review on chemical process fault detection and diagnosis, 6th International Symposium on Advanced Control of Industrial Processes (AdCONIP) (2017). https://doi.org/10.1109/ adconip.2017.7983824. 
[7] Y.-J. Park, S.-K. S. Fan, C.-Y. Hsu, A Review on Fault Detection and Process Diagnostics in Industrial Processes. Processes, 8(9), 1123 (2020). https: //doi.org/10.3390/pr8091123.

[8] L. Dai. Singular Control Systems. Lecture Notes in Control and Information Sciences. Springer-Verlag. Berlin, 1989.

[9] A. Kumar and P. Daoutidis, Control of nonlinear differential algebraic equation systems. Chapman \& Hall CRC, 1999.

[10] G. R. Duan. Analysis and Design of Descriptor Linear Systems. Springer 2010.

[11] M. Darouach, M. Boutayeb, Design of observers for descriptor systems, IEEE Transactions on Automatic Control, vol. 40, pp. 1323-1327 (1995). https://doi. org/10.1109/9.400467.

[12] D. Koenig, Unknown Input Proportional MultipleIntegral Observer Design for Linear Descriptor Systems: Application to State and Fault Estimation, IEEE Transactions on Automatic Control, Vol. 50, No. 2 (2005). https://doi.org/10.1109/TAC.2004. 841889.

[13] Z. Gao, S. X. Ding, Y. Ma, Robust fault estimation approach and its application in vehicle lateral dynamic systems, Optimal Control Applications and Methods, vol. 28, no. 3, pp. 143-156 (2007). https: //doi.org/10.1002/oca.786.

[14] Z. Wang, Y. Shen, X. Zhang, Q. Wang. Observer design for discrete-time descriptor systems : An LMI approach, Systems and Control Letters, 61(6) :683687 (2012). https://doi.org/10.1016/j.sysconle.2012. 03.006

[15] M. Darouach, On the functional observers for linear descriptor systems, Syst. Control Lett., vol. 61, no. 3, pp. 427-434 (2012). https://doi.org/10.1016/j. sysconle.2012.01.006.

[16] Q. Jia, H. Li, Y. Zhang, X. Chen Robust Observerbased Sensor Fault Reconstruction for DiscreteTime Systems via a Descriptor System Approach, International Journal of Control, Automation and Systems 13(2):1-10 (2015). https://doi.org/10.1007/ s12555-014-0098-0

[17] G.-L. Osorio-Gordillo, M. Darouach, C.-M. AstorgaZaragoza, L. Boutat-Baddas, Fault diagnosis for discrete-time descriptor linear systems, International Federation of Automatic Control, vol. 48, no. 21, 1238-1243 (2015). https://doi.org/10.1016/j.ifacol. 2015.09.695.

[18] Z. Wang, M. Rodrigues, D. Theilliol, Y. Shen, Fault estimation Filter design for discrete-time descriptor systems, IET Control Theory and Applications, Institution of Engineering and Technology, 9(10), pp.1587-1594 (2015). https://doi.org/10.1049/ iet-cta.2014.0641.

[19] Z. Gao, Fault estimation and fault tolerant control for discrete-time dynamic systems, IEEE Transactions on Industrial Electronics, 62(6), 3874-3884 (2015). https://doi.org/10.1109/TIE.2015.2392720.

[20] I. Haj Brahim, M. Chaabane, G. Hashim and D. Mehdi, Sensor Fault and State Estimation for Continuous-Time Descriptor Linear Systems: an LMI Approach. Proceedings of the 2016 5th International Conference on Systems and Control, Cadi Ayyad University, Marrakech, Morocco, May 25-27 (2016). https://doi.org/10.1109/ICoSC.2016. 7507057.

[21] J. Zhang, F. Zhu, J. Li, X. Li, Discrete-time linear descriptor system unknown input observer design: an auxiliary output-based approach, International Journal of Control, Automation and Systems, 15(6), 2599-2607 (2017). https://doi.org/10.1007/ s12555-016-0611-8.

[22] K. Bouassem, A. El Assoudi, J. Soulami, E. El Yaagoubi, Unknown Input Observer Design for a Class of Linear Descriptor Systems. The $3^{\text {rd }}$ International Conference of Computer Science and Renewable Energies (ICCSRE'2020), Agadir, Morocco, December 22-24, 2020. https://doi.org/10.1051/e3sconf/202122901019.

[23] S. Boyd, L. E. Ghaoui, E. Feron, V. Balakrishnan, Linear Matrix Inequalities in Systems and Control Theory, Philadelphia, PA: SIAM (1994). https://doi. org/10.1137/1.9781611970777. 